\title{
Integrated Modelling of an Unmanned High-Altitude Solar-Powered Aircraft for Control Law Design Analysis
}

\author{
Andreas Klöckner, Martin Leitner, Daniel Schlabe, Gertjan Looye
}

\begin{abstract}
Solar-powered high-altitude unmanned platforms are highly optimized and integrated aircraft. In order to account for the complex, multi-physical interactions between their systems, we propose using integrated simulation models throughout the aircraft's life cycle. Especially small teams with limited ressources should benefit from this approach. In this paper, we describe our approach to an integrated model of the Electric High-Altitude Solar-Powered Aircraft ELHASPA. It includes aspects of the environment, flight mechanics, energy system, and aeroelasticity. Model variants can be derived easily. The relevant parts of the model are described and the model's application is demonstrated.
\end{abstract}

\section{Introduction}

Solar-powered aircraft have received increasing interest by the scientific and industrial community in the past fifty years. The subject of early solar flight is summarized in depth by Boucher[3]. Voit-Nitschmann[20] and Noth[14] extend the overview and the latter also includes a list of flown solar aircraft with their basic characteristics. Recent developments of the Solar Impulse[19] and the QinetiQ Zephyr[15] prove that the technology is ready to allow for continuous solar-powered flight.

However, solar-powered and high-altitude aircraft rely on highly optimized aircraft design. This results in high lift configurations, fragile light-weight construction and restrictive power reserves. These aircraft are thus constantly operated at their physical limits. All aspects of the systems work in a highly integrated manner and influence one another in a way, which is difficult to predict. Examples of the interactions can be found between flight dynamics and the energy system or between the flight dynamics

Andreas Klöckner, Martin Leitner, Daniel Schlabe, Gertjan Looye

German Aerospace Center, Robotics and Mechatronics Center, Institute of System Dynamics and Control, Münchner Straße 20, D-82234 Oberpfaffenhofen-Weßling, e-mail: andreas . kloeckneredlr.de 
and the elastic modes of the aircraft. When an autopilot feedback loop is introduced, the system becomes even more complex. Fig. 1 summarizes the interactions to be taken into account, when developing or operating high-altitude solar-powered aircraft.

Fig. 1 A multitude of interactions must be taken into account, when developing or operating high-altitude solar-powered aircraft. The modules of the simulation are shown together with the mutual influences. Arrows indicate causality. Examplary couplings are labelled with the type of influence. Dashed arrows indicate interactions, which are not covered by the present model.

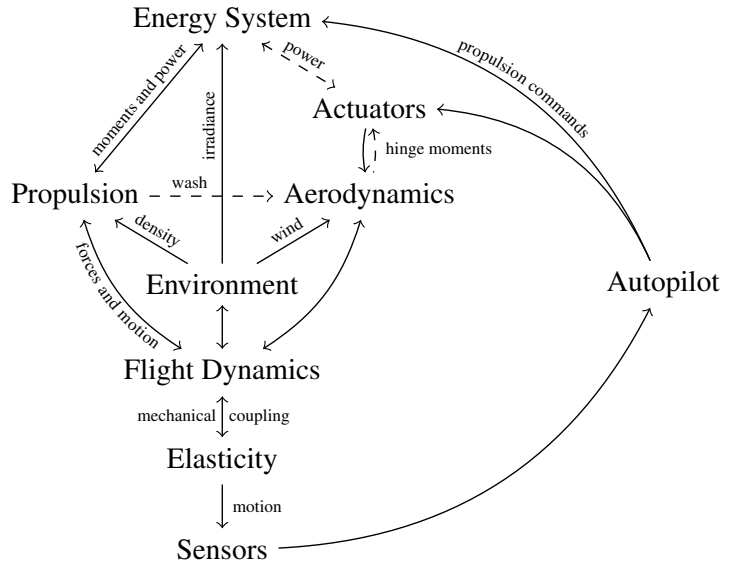

The high level of integration of all these modules renders the development, simulation and operation of high-altitude solar-powered aircraft a difficult task. Thus, we propose to use integrated models during the whole life cycle of solar aircraft. They provide faster and more accurate feedback to the design team of the aircraft than separate models, which are traditionally used. Refined versions of the models can later be used for more realistic simulation, training, control design and hardware-in-the-loop tests. An integrated model structure finally also provides consistent modelling of all parts of the system and for all design and operational analyses to be carried out. These features are especially valuable for small teams with limited ressources.

In order for this approach to work, the modelling technique must be suitable for a variety of physical fields. The resulting models must be fast enough for closed-loop simulation. Also, model versions of differing level of detail and reduced degrees of freedom must be derived with little effort. The approach of the present study makes use of Modelica [6] and the Flight Dynamics Library [12] to solve these difficulties.

Modelica is an open-source modelling standard, explicitly designed for multidisciplinary and multi-physics modelling and simulation. Its equation-based approach also allows for automatically generating model derivations such as linear state-space or non-linear dynamic inverse models. The model equations can be exported to Ccompiled code, allowing for fast simulation and integration in Simulink S-functions or embedded systems using the Functional Mockup Interface [2].

The DLR Flight Dynamics Library provides a great variety of basic aircraft models as well as environmental models necessary for six degrees of freedom (6DOF) and aeroelastic simulation of conventional aircraft. Positions can be expressed in the World Geodetic System (WGS84) and the rotation of the earth is taken into account. 
Detailed models of the magnetic and gravitational fields of the earth are included in the library as well as standard atmosphere data and wind simulation.

A major advantage of the Flight Dynamics Library is that it extensively uses standard Modelica mechanical connectors, such as MultiBody frames. This allows to connect any additional model with force or position interfaces to the airframe by a standardized interface without changing the model equations. Interactions other than mechanical connections are typically realized with Modelica's "outer" models providing global functions for the environmental properties. Buses are used for internal control signals. The present model makes use of these techniques and keeps up the interfacing philosophy.

Previous work on this subject has been published in [11]. In this paper, we enhance the model with elastic modes and an autopilot to gain a more holistic view of the system. Thus, all interactions as shown in Fig. 1 are taken into account, except for the dashed lines.

In the following sections, we first give an introduction to the solar aircraft used in our study. We then introduce the overall model structure in section 3. Section 4 summarizes the main components of the integrated model. In section 5 , we briefly present selected appplications for the model and section 6 provides conclusions and an outline of future work.

\section{The ELHASPA Aircraft}

In this paper, the Electric High-Altitude Solar-Powered Aircraft (ELHASPA) serves as an example for the developed modelling approach. It is developed and built by a consortium including the German Aerospace Center's (DLR) Robotics and Mechatronics Center (RMC) and several industrial partners. The unmanned aircraft is intended to advance technologies and test applications for continuous high-altitude solar-powered flight. A design model of the aircraft is shown in Fig. 2.

Fig. 2 The ELHASPA aircraft has a wing span of $23 \mathrm{~m}$ and a mass of $100 \mathrm{~kg}$. It has two separate avionics systems in its two fuselages and is intended to advance technologies and test applications for continuous high altitude solar flight.

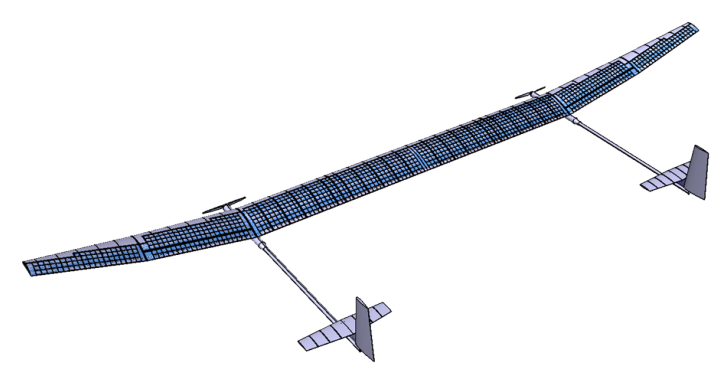


ELHASPA's light-weight carbon fiber construction has a span width of $23 \mathrm{~m}$, a wing area of $25 \mathrm{~m}^{2}$ and a total mass of $100 \mathrm{~kg}$. It is designed to fly at speeds of $6 \mathrm{~m} / \mathrm{s}$ to $15 \mathrm{~m} / \mathrm{s}$ and in stratospheric altitudes of more than $15 \mathrm{~km}$. It has two separate fuselages with identical energy and avionics systems. The two ailerons and the all-movable elevators and rudders are independently steerable.

\section{Integrated Model Structure}

While Modelica was chosen as the basic modelling environment, simulation and autopilot development is performed in Simulink. All model components and their interactions illustrated in Fig. 1 are therefore Modelica-based except for the autopilot. The connections are mostly realized by the use of Modelica's outer models and Modelica's standard mechanical connectors. The model is then exported to Simulink and connected to the autopilot. Sensor and control data are passed as regular signals. The overall model structure is shown in Fig. 3.

The model consists of three main parts. The aircraft model is separated from the environment model, such that environmental information is globally available to all submodules and possibly multiple aircraft. Data is exchanged between the aircraft and the environment modules by Modelica's "outer" models, providing global functions and variables. The autopilot model is again separated from the plant model and can be implemented in Simulink, Modelica or any suitable modelling language. It is connected to the plant by mere signal flows, making possible this multi-tool approach.

The aircraft model is driven by a central airframe module, which implements the equations of motion. All modules requiring or generating position, attitude, force or moment information are connected to the airframe by physical "MultiBody" connectors. This includes aerodynamics, sensors, propulsion and aeroelasticity modules. The propeller of the propulsion system is driven by a rotational flange connector, which is connected to the motor flanges of the energy system. Actuator models shape the command signals for the aerodynamic's control surface deflections.

A variety of base models is already available in the Flight Dynamics Library. The modularity of the library is maintained by defining additional modules and modular upgrades to existing modules. For example, the atmospheric effects on radiation are contained in a model called "IrradianceUpgrade", which is then overloaded on the existing standard atmosphere model. All modules can be seamlessly interchanged with the modules already provided by the Flight Dynamics Library.

The following section summarizes the modules particular to the ELHASPA case. 


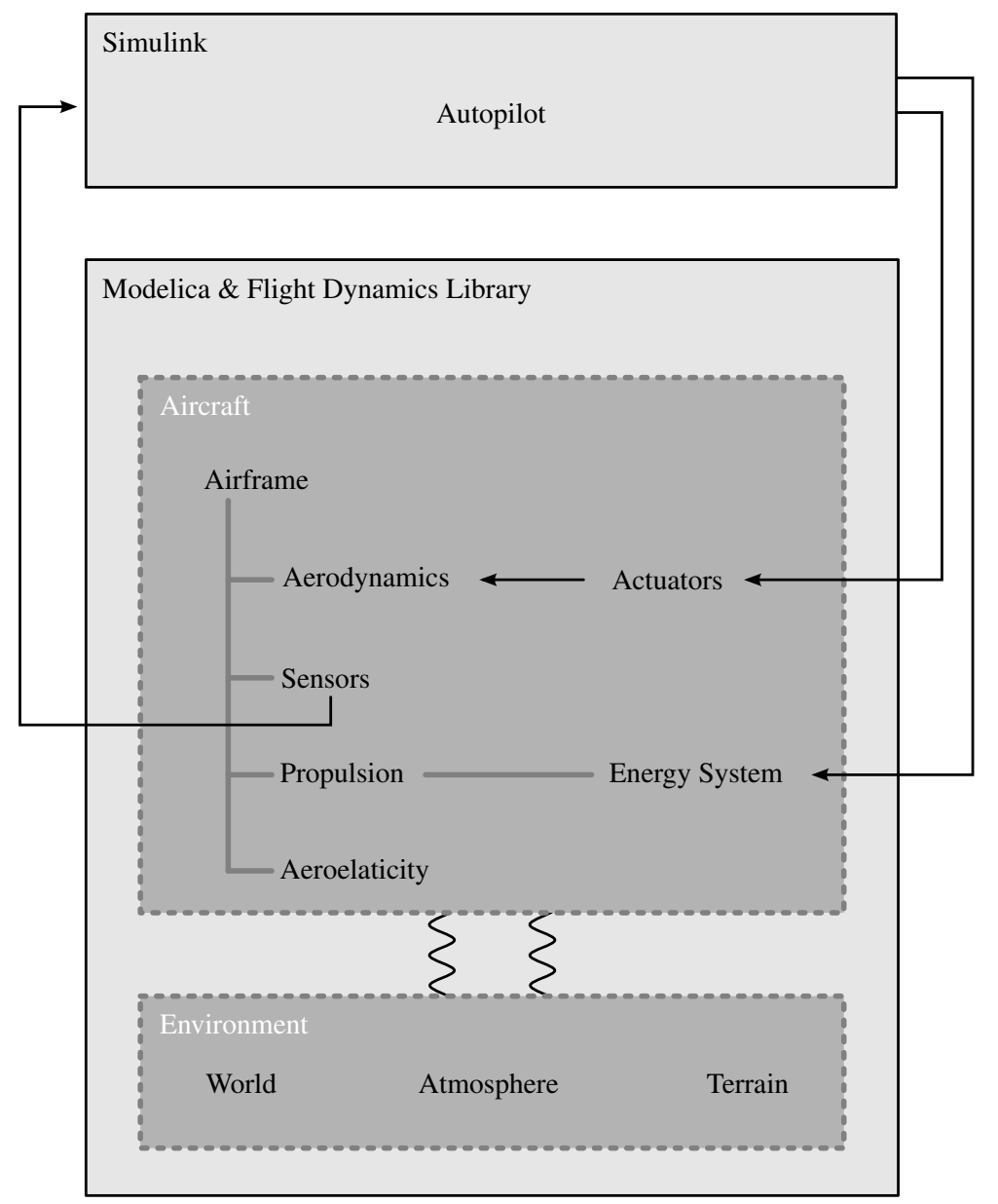

Fig. 3 The overall model is comprised of a main and a controller module, realized in Modelica and Simulink respectively. Interactions between aircraft and environment are managed by "outer" models in Modelica $(\checkmark)$, whereas internal aircraft components are mostly linked by physical connectors $(-)$. The autopilot is connected to the aircraft model using regular signal exchange $(\longrightarrow)$.

\section{Components of the ELHASPA Model}

The main parts of the ELHASPA model are described briefly in this section. This includes the radiation calculation within the environment model, the energy system, the propulsion and the aerodynamics modules of the aircraft. Special attention is paid to the unsteady aerodynamics and aeroelasticity. The autopilot is described in a separate paper [9]. 


\subsection{Environment}

The environment model provides reference systems and gravity models for the world and atmospherical quantities relevant to flight mechanics such as the wind, static pressure and air density. For solar aircraft, it is extended with additional components to simulate the position of the sun and the resulting solar irradiance as well as atmospheric refraction (ray deviation) and extinction (ray attenuation) of the solar radiation. This allows to simulate day/night cycles including a fine resolution at the horizon and attenuation of the solar radiation for altitudes close to the earth's surface.

The calculation of the sun's position is derived from Modelica's Satellite Dynamics library and is build on a simplified analytical sun model by Schlyter [18]. This model implements the sun as a regular earth satellite on an elliptical orbit with changing orbital elements. The mean solar irradiance is given by $I_{0}=1366 \mathrm{~W} / \mathrm{m}^{2}$ and varies slightly with the distance between the sun and the earth.

The atmospheric refraction $R$ is modelled to deviate rays of sunlight from the direct path according to Saemundsson [17]. The atmospheric extinction is modelled using the relative airmass $A M$ according to Young [22] and Honsberg and Bowden [8]. These models are valid for operation in relatively low altitudes. For actual stratospheric flight simulation, additional effects of the solar spectrum and modified extinction calculations have to be included in the models.

The modelled irradiation characteristics are depicted in Fig. 4. A more detailed description of the modifications to the standard Flight Dynamics Library models can be found in [11].

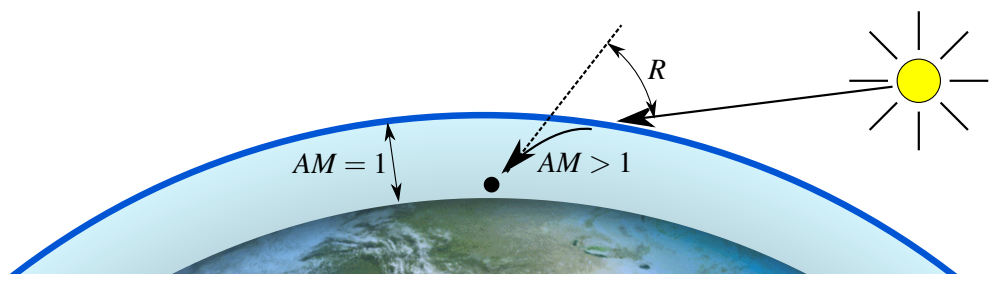

Fig. 4 The additional environmental model includes simulation of the sun location, the atmospheric refraction $R$ and the atmospheric extinction as a function of the relative airmass $A M$.

\subsection{Energy System}

The energy system consists of independent electrical systems for each fuselage of the aircraft. Each side contains a solar energy generation block, a battery pack, an electric motor, and further technical loads. The batteries and the solar generation block are directly connected to the high voltage power bus. The motor is driven by a motor controller. The low voltage technical loads are powered by a voltage 


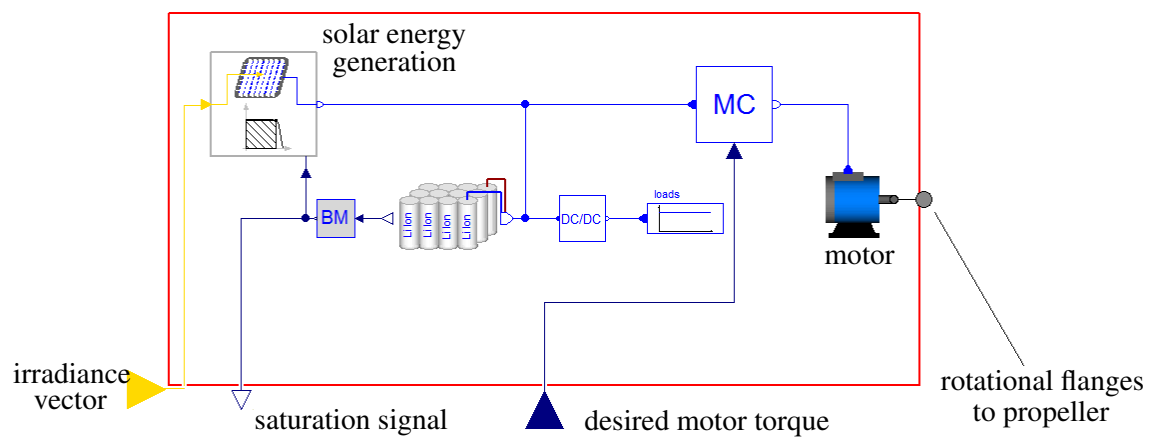

Fig. 5 One half of the energy system model including solar cells driven by the incoming irradiance, batteries, a motor controller (MC) driving a motor and technical loads. The battery manager (BM) provides feedback on the batteries' state of charge.

converter. A battery manager provides feedback on the current state of charge to the solar energy generators. Fig. 5 depicts one side's electrical system.

Each solar generation block contains several solar panels. Each solar panel has a dedicated position on the aircraft structure and thus is exposed to an individual irradiance. The entire panel is modelled by means of one diode connected in parallel to a current source. The source generates a current proportional to the solar irradiance. An additional maximum power point tracker sets the operating voltage of the panels such that the maximum power is retrieved. All outputs of the maximum power point trackers are then connected in parallel to the main power bus.

The battery pack is modelled by a voltage source and an inner resistance, which are both stored in look up tables as functions of the state of charge and current flow. The battery's state of charge is obtained by integrating the electric current. An additional battery manager is needed in order to prevent the battery from overload. To this end, the battery manager further reduces the voltage of the solar panels in such a way, that no surplus power is generated. The internal saturation signal is provided for use in the control loops. It indicates the amount of currently unused solar power.

The electrical motor is the major consumer of electrical power and drives the aircraft propeller through a Modelica rotational flange. The model consists of an electromagnetic force, which ideally transforms electrical energy into mechanical energy, an electrical resistor to consider copper losses, and a variable friction at the mechanical side to incorporate iron losses. The motor torques are commanded through a control signal.

Technical loads like computers, servos, and communication devices are modelled by a fixed electrical resistance. These loads are powered by a voltage converter considering constant-efficiency as well as fixed losses of the converter. 


\subsection{Propulsion}

The aircraft is equipped with two fixed-pitch propellers. Their characteristics have been estimated with the help of a Blade Element Method provided by the JavaProp software [7]. The resulting thrust force and the torque on the propeller shaft have been calculated for a grid of about 16000 points at different propeller turn rates, aircraft airspeeds and altitudes. The propeller is driven through a Modelica standard rotational connector, which allows to connect arbitrary motor models to the propeller model, such as direct speed inputs or detailed motor models. The data is interpolated at runtime to yield the current propeller thrust and shaft torque. The resulting forces and moments are applied through a MultiBody connector to the airframe.

\subsection{Steady Aerodynamics}

The baseline steady aerodynamics of the aircraft are modelled using the Athena Vortex Lattice (AVL) software [4], a potential flow simulation for thin airfoils. The estimation is augmented with airfoil data obtained from Xfoil [5]. These routines provide fast aerodynamic estimation and they do not require extensive modelling. Thus, the aerodynamic methods described here are capable of easily following design changes and providing feedback to the design process in turn. Qualitative assessment based on the pilot's experience from training and the first test flight indicates a good match between simulation and real flight.

The aerodynamic model is composed of the main wing, two horizontal, and two vertical stabilizers. It is depicted in Fig. 6 along with the AVL coordinate system. The model contains 1680 panels and six independently actuated control surfaces. The differing axes definition from standard notation is handled by an extension to the Flight Dynamics Library's base models.

Fig. 6 ELHASPA's vortex lattice mesh contains 1680 panels for the main wing, horizontal and vertical stabilizers and six independently actuated control surfaces.

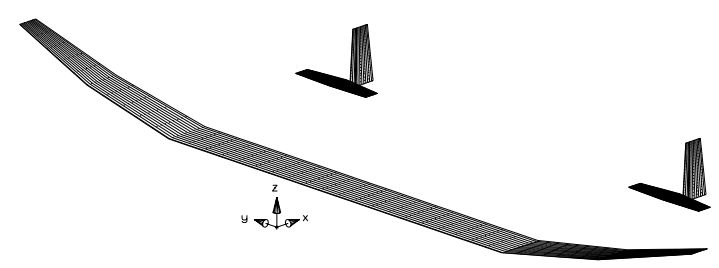

As the vortex lattice method is a detailled but mostly linear modelling tool, all surfaces are augmented with Xfoil polars. The polars provide nonlinear drag effects, which lead to a stop of the simulation in case of stall.

Since the computation of the flow field within AVL cannot be carried out in real time, the aerodynamic model is reduced to a polynomial approximation of the AVL model. To this end, a number of 10000 points are calculated in the relevant flight envelope. The resulting aerodynamic coefficients are fitted in a least squares sense 
to multi-dimensional polynomials $\mathrm{p}\left(x_{1}, x_{2}, \ldots\right)$ in the aerodynamic angles $(\alpha$ and $\beta$ ), the rotational rates $(p, q, r)$ and the control surfaces $\left(\delta_{a}, \delta_{e}, \delta_{r}\right)$. These polynomials also cover coupling effects of the form $x_{1}^{m} \cdot x_{2}^{n}$. The output equations from the above procedure have the following form:

$$
\begin{aligned}
C_{L} & =\mathrm{p}\left(\alpha, q, \delta_{a}, \delta_{e}\right) & & \text { (Lift coefficient) } \\
C_{D} & =\mathrm{p}\left(\alpha, q, \delta_{a}, \delta_{e}\right) & & \text { (Drag coefficient) } \\
C_{Y} & =\mathrm{p}\left(\beta, p, r, \delta_{a}, \delta_{r}\right) & & \text { (Side-force coefficient) } \\
C_{l} & =\mathrm{p}\left(\beta, p, r, \delta_{a}, \delta_{e}, \delta_{r}\right) & & \text { (Rolling moment coefficient) } \\
C_{m} & =\mathrm{p}\left(\alpha, q, \delta_{a}, \delta_{e}\right) & & \text { (Pitch moment coefficient) } \\
C_{n} & =\mathrm{p}\left(\beta, p, r, \delta_{a}, \delta_{e}, \delta_{r}\right) & & \text { (Yaw moment coefficient) }
\end{aligned}
$$

\subsection{Unsteady Aeroelasticity}

By combining a traditional nonlinear rigid body flight dynamics model with linear aeroelastic dynamics, the equations of motion of the flexible aircraft can be derived. These equations combine the rigid body motion of the aircraft and the elastic deflections with respect to a body fixed system. Details may for example be found in Waszak and Buttrill [21]. After a couple of simplifying assumptions and constraints, the nonlinear rigid body and flexible equations can be decoupled and are then available in their traditional form.

The flexible part of the integrated model considers the representation of the aircraft by a chosen number of linear elastic modes obtained through modal analysis of the underlying condensed structural model of the aircraft. Rigid motion is defined by six rigid body modes corresponding to the six degrees of freedom of a body reference frame fixed to the center of gravity in three-dimensional space. The classic linear elastic equations of motion are then generalized by the elastic modes and driven by the corresponding generalized coordinates.

In the Modelica Flight Dynamics Library arbitrary structural forces can easily be incorporated by flexible connectors. Structural grid indices are used to specify the location of the modal displacements within the modal basis to obtain the corresponding generalized flexible forces. Updating the resulting forces and moments about the rigid motion reference frame then simply becomes a matter of incorporating the momentary structural grid displacements and rotations.

Rigid body modes in the body fixed reference frame are added to the modal basis so that the nonlinear rigid body and elastic equations of motion are coupled after generalizing a set of distributed aerodynamic forces. In the presented case unsteady aerodynamic forces acting on the structural frame have been calculated using the so called doublet lattice method (DLM) (see e.g. Albano and Rodden [1]). DLM aerodynamic forces are derived from an aerodynamic panel model of the lifting surfaces. The result are constant complex valued matrices of aerodynamic influence coefficients (AICs) relating the harmonically varying downwash on each of those 
panels to the pressure difference across each of the other panels. Obtaining the aerodynamic forces thus becomes a matter of determining the downwash due to rigid body motion and elastic deflections and multiplying the resulting panel pressures with the respective area of the panel [10].

Fig. 7 DLM panel mesh of the ELHASPA aircraft. The red dots represent the grid points of the condensed structural model.

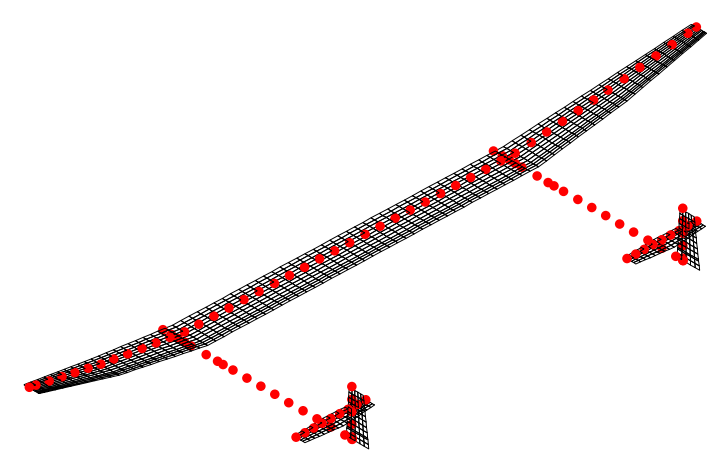

To be compatible to the generalized equations of motion, three subsequent postprocessing steps are required. First the DLM coefficients are available as complex valued matrices in frequency domain only and have to be transformed to the time domain. Next the aerodynamic grid is splined to the structural grid by a conventional beam spline as for example implemented in the commercial aeroelastic solvers of Msc's NASTRAN [13], so that rigid and flexible motion can be related to the applied downwash as well as integrated aerodynamic pressures to structural forces. Roger's rational function approximation (see [16]) was therefore applied which allows conversion to time domain by a simple inverse Laplace transformation, but also leads to additional differential equations to capture the phase shift towards the corresponding quasi-steady forces. In a second step, the generalization of the so obtained real valued unsteady aerodynamic coefficients using the modal basis of rigid and flexible modes leads to rigid and elastic forces as well as coupling forces between the former two. The coupling forces provide the influence from rigid body motion onto the flexible structure and vice versa such that the aircraft's elastic structural behaviour is fully integrated in the simulation model. Additional modes were added to the modal basis to account for excitation by control surface deflections and turbulence.

\section{Selected Applications}

The integrated model is used throughout the development and commissioning of the ELHASPA aircraft. Valuable feedback to the design team include aerodynamic parameter studies and flutter estimations. In this section, two selected applications are introduced. The preliminary analysis of a control law is illustrated by comparing 

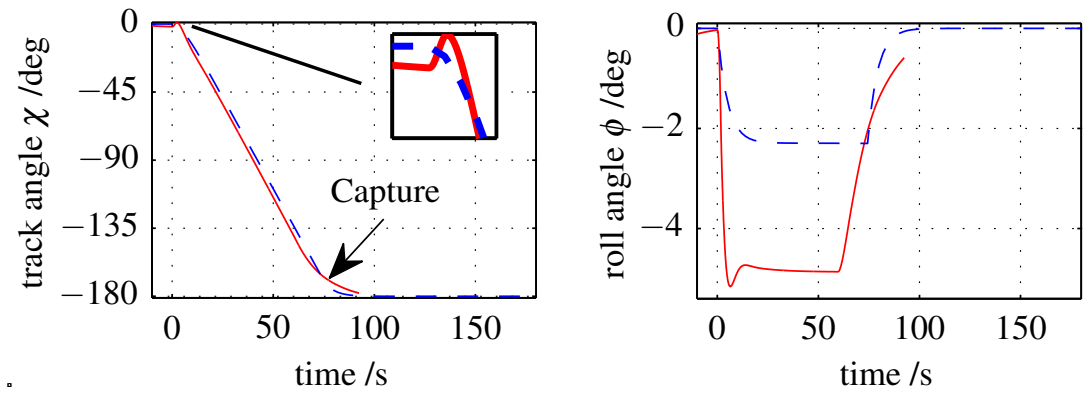

Fig. 8 The track and roll angle plots for the point mass inverse model (- - ) and the controlled flexible model (-).

controlled flight of the full model to a reduced mission simulation. The second example illustrates the influence of wing flexibility on the harvested solar energy.

The reduced mission simulation has been previously introduced in [11]. In a first step, the ELHASPA model is reduced to a point mass model neglecting elasticity and the energy system. For this reduced model, a non-linear dynamic inverse model is automatically generated using Modelica. The inverse model directly receives the trajectory as inputs and computes all relevant aircraft states from it. The reduced mission simulation is approximately 50000 times faster than realtime. The results are compared to a second simulation. It includes the fully flexible ELHASPA model and the autopilot being developed for the aircraft (see [9] for details). The results are compared for a turn of $180^{\circ}$ taking approximately 100 seconds.

Figure 8 shows the track angle $\chi$ and the roll angle $\phi$ for the described maneuver. The track angle develops a similar constant turn rate in both cases. However, the transient behavior is more detailed in the full model case. The autopilot reacts quicker to the command and uses an acquire-and-hold procedure to capture the new track angle command.

The simulated roll angle is different for both cases: The inverse model illustrates the ideal kinematic response of ELHASPA to the track angle change. The controlled model shows actual dynamic behavior as expected from the controlled aircraft. During the turn, the controlled model builds up an adverse sideslip angle. This must be compensated for by a higher roll angle.

A similar effect can be observed in data from the first manual test flights. Note, that they have not been optimized for model validation. Figure 9 shows a turn of approximately $220^{\circ}$ taking about 40 seconds. The trajectory data is used to steer an inverse point mass model taking into account steady aeroelastics. First, the reconstructed sideslip angle is used. The model follows the given trajectory exactly and the good agreement in terms of roll angle are an indicator of a good lateral simulation model. If the same trajectory is simulated maintaining zero sideslip angle, the necessary roll angle is visibly decreased. This is according to expectations and confirms the effect found with the controlled simulation. 

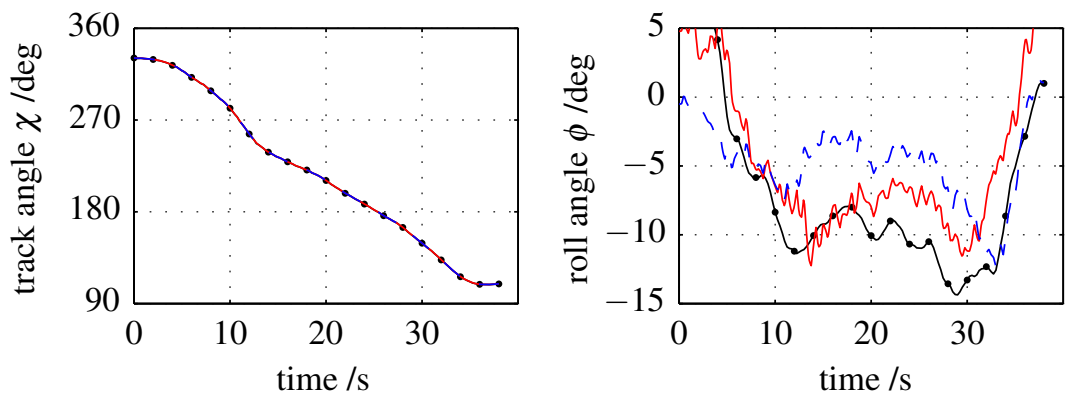

Fig. 9 A trajectory from flight experiments $(\bullet-)$ is re-simulated using an inverse point mass model with the reconstructed (-) and with zero (- ) sidelip angle.

To show the response of the model to unsteady wind profiles, a '1-cosine' gust profile with a short gust reference length is applied to the fully flexible model. The gust gradually applies downwash to the wing while travelling downstream. The profile and the rigid body part of the model response are shown in Fig. 10. The aircraft response is corrected by the trim angle of attack of approximately $-7^{\circ}$, which is due to the wing's high angle of incidence.

The initial response to the gust is a mere change in sensed angle of attack $\alpha$. This change is then also converted to a kinematic pitch attitude. The dynamics of the aircraft are strongly damped and return slowly to their trim state. Minor oscillations can still be observed, right after the gust has passed.

Figure 11 illustrates the flexible response of ELHASPA's lifting surfaces to the '1-cosine' gust. It also illustrates the flexibility influence on a solar panel attached to the right wing tip. Each solar panel is fixed relative to a single structural grid. By using flexible instead of rigid connector blocks, the position and orientation of the grid points and hence the wing's deformation is taken into account when calculating the irradiance.

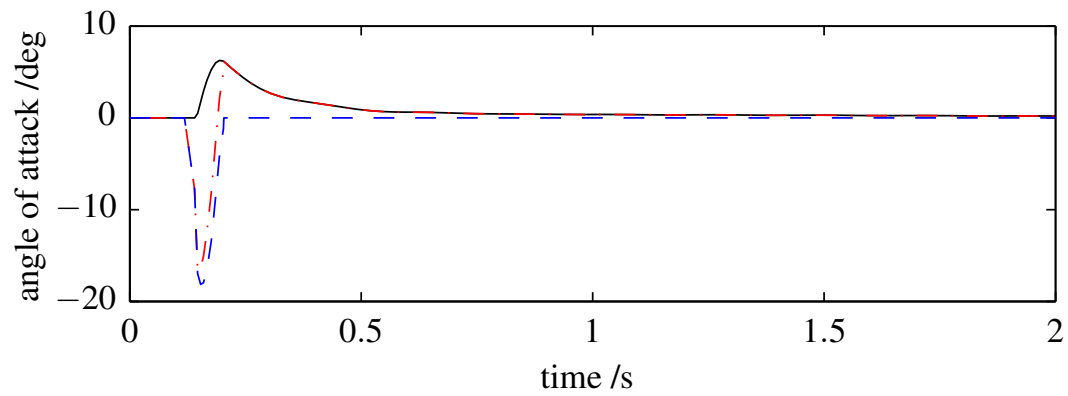

Fig. 10 The applied '1-cosine' gust profile (--) is depicted in terms of angle of attack. The rigid body part of the response is shown by the kinematic and aerodynamic angles of attack $\alpha_{F}(-)$ and $\alpha(---)$. The aircraft angles of attack are corrected by the trim state. 

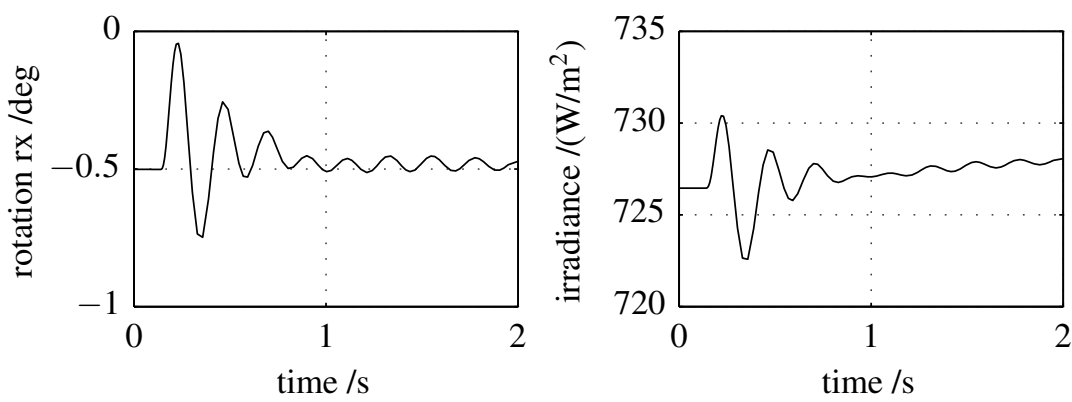

Fig. 11 The flexible response to the '1-cosine' gust is illustrated by the behavior of a structural grid point and the attached solar panel. The pair is located at the outward right wing side. The rotation rx about the $\mathrm{x}$-axis is measured in loads axes of the structural grid with positive $\mathrm{x}$ is downstream. The resulting variation in irradiance can be seen in the right graph.

ELHASPA's elastic response is dominated by the first symmetric bending mode, which bends the central section between the engines upwards and rotates the outer wing tips downwards. In trimmed flight, this is indicated by the negative $\mathrm{x}$-axis rotation of the outward structural grid on the right wing tip as shown in the graph on the left hand side. The gust then hits the lifting surface leading to an inital upward deflection followed by damped oscillations. As can be seen in the graph on the right side, these high frequency oscillations in the grid orientation manifest themselves in a corresponding change of irradiance on the solar panel. The low frequency rigid body motion initiated by the coupling with the elastic model can also be observed.

\section{Conclusions}

We have presented an integrated modelling approach for the Electric High-Altitude Solar-Powered Aircraft ELHASPA. It meets all design requirements: The relevant multi-physics interactions between flight dynamics, energy system, aeroelastics and the environment are covered. The system is modular and can be extended easily to cover remaining aspects. The approach provides a consistent modelling scheme of all aspects of the aircraft. The simulation is sufficiently fast for closed-loop simulations. Model variants for most imaginable applications can be derived easily, yielding also extremely fast reduced variants.

The model is used in the overall design, flight test and operation process. This includes application on different operating systems such as realtime embedded systems and desktop computers. The use of a single integrated model proves efficient for a small project team.

Future work will concentrate on validating and extending the model. All modules will be validated and updated with flight and ground tests. Results of recently conducted load tests will be used to validate the elastic model. Additional modules will 
be included to allow for high-fidelity simulation of longer flights in high altitudes, such as temperature effects and servo energy consumption. The model will also be exploited for the continuing development and operation, especially of the autopilot and control laws as well as mission planning and management.

\section{References}

[1] Albano E, Rodden W (1969) A doublet-lattice method for calculating lift distributions on oscillating surfaces in subsonic flow. AIAA Journal 7:279-285

[2] Blochwitz T, Otter M, Arnold M, Bausch C, Clauß C, Elmqvist H, Junghanns A, Mauss J, Monteiro M, Neidhold T, et al (2011) The functional mockup interface for tool independent exchange of simulation models. In: Modelica'2011 Conference, March, pp 20-22

[3] Boucher R (1984) History of solar flight. AIAA paper pp 84-1429

[4] Drela M, Youngren H (2004) Athena vortex lattice. online at http:// raphael.mit.edu/avl, URL http://raphael.mit.edu/avl

[5] Drela M, Youngren H (2008) Xfoil - subsonic airfoil development system. online at http://raphael.mit.edu/xfoil, URL http://raphael. mit.edu/xfoil

[6] Fritzson P (2004) Principles of object-oriented modeling and simulation with Modelica 2.1. Wiley-IEEE Press, URL http: //books.google.de/books? id=I zqY8AbzlrAC\&print sec= frontcover\&dq=Principlestoftobject-oriented+ modeling+and+simulation+with+Modelica+2.1\&hl= de\&sa $=X \& e i=Q k k N T 9 E f z-C z B q O p z J w E \& v e d=0$ CEYQ 6AEwAA\# $\mathrm{v}=$ onepage $\& \mathrm{q} \& \mathrm{f}=\mathrm{false}$

[7] Hepperle M (2010) JavaProp Users Guide

[8] Honsberg C, Bowden S (2010) Pvcdrom. online at http://www. pveducation.org/pvcdrom/properties-of-sunlight/ air-mass, URL http://www.pveducation.org/pvcdrom/ properties-of-sunlight/air-mass

[9] Kastner N, Looye G (2013) Generic tecs based autopilot for an electric high altitude solar powered aircraft. In: CEAS EuroGNC, CEAS, Delft, The Netherlands, submitted for publication

[10] Kier TM, Looye GHN (2009) Unifying manoeuvre and gust loads analysis models. IFASD paper

[11] Klöckner A, Schlabe D, Looye G (2012) Integrated simulation models for high-altitude solar-powered aircraft. In: AIAA Modeling and Simulation Technologies Conference, AIAA, Minneapolis, Minnesota, aIAA 2012-4717

[12] Looye G (2008) The new dlr flight dynamics library. In: Proceedings of the 6th International Modelica Conference, vol 1, pp 193-202

[13] MSC (2004) Nastran Version 68 Aeroelastic Analysis User's Guide 
[14] Noth A (2008) History of solar flight. Aircraft \& spacecraft systems design lecture notes, Autonomous Systems Lab, ETH Zürich, Switzerland

[15] QinetiQ Group, PLC (2010) Zephyr UAV. online at http://www2. qinetiq.com/home_farnborough_airshow/unmanned_ air_systems/zephyr.html, URL http://www2.qinetiq. com/home_farnborough_airshow/unmanned_air_systems/ zephyr.html

[16] Roger K (1977) Airplane math modeling methods for active control design. AGARD

[17] Saemundsson T (1986) Atmospheric Refraction. Sky And Telescope 72:70

[18] Schlyter P (2011) How to compute planetary positions. online at http://stjarnhimlen.se/comp/ppcomp.html, URL http:// st jarnhimlen.se/comp/ppcomp.html

[19] Solar Impulse S (2011) Solar impulse - around the world in a solar airplane. online at http://solarimpulse.com/, URL http://solarimpulse. $\mathrm{com} /$

[20] Voit-Nitschmann R (2001) Solar- und Elektroflugzeuge - Geschichte und Zukunft. In: Jahrbuch aus Lehre und Forschung der Universitt Stuttgart, Universitt Stuttgart, pp 88-99

[21] Waszak MR, Buttrill CS (1992) Modeling and model simplification of aeroelastic vehicles: An overview. Tech. rep., NASA

[22] Young AT (1994) Air mass and refraction. Applied optics 33(6):1108-1110 